\title{
A NOTE ON THE SEPARABILITY OF CANONICAL INTEGRATIONS OF LIE ALGEBROIDS
}

\author{
David Martínez TORRes
}

\begin{abstract}
We find criteria to constrain the lack of separability of canonical integrations of Lie algebroids. As an application we deduce separability of canonical symplectic integrations of Poisson structures on real surfaces, and Poisson structures underlying generalized complex structures of even type on four dimensional manifolds, the latter including holomorphic Poisson structures on (smooth) complex surfaces. Separability can then be used to describe further properties of these canonical integrations.
\end{abstract}

\section{Introduction}

The canonical integration $\mathcal{G}(A) \rightrightarrows M$ of an integrable Lie algebroid $A \rightarrow M-M$ second countable and Hausdorff- is a Lie groupoid which is second countable, whose 1-connected source and target fibers are Hausdorff, but which may not be Hausdorff.

There are very good reasons to allow non-separable Lie groupoids, the main ones coming from the role of homotopy groupoids in foliation theory. Often a groupoid is constructed out of some geometric structure, and invariants of the groupoid -and hence of the original structure- such as cohomology do not rely on separability.

Lie groupoids can be thought of as manifolds with local symmetries. Sometimes they come naturally endowed with structures compatible with the local symmetries -multiplicative structures- as it is the case for canonical integrations of (twisted) Poisson, (twisted) Dirac, and Jacobi manifolds [5, 4, 6, 11, 3]. Therefore these Lie groupoids are a distinguished class of (twisted) symplectic, (twisted) presymplectic, and contact manifolds respectively, and thus worth being studied from the point of view of these geometries. But one cannot even do a reasonable manifold theory without separability. Hausdorffness allows the use of all tools from differential topology. An example of this appears in [10], where separability plays a vital role in the geometric proof of Conn's linearization; another one is corollary 1 below. In light of the previous discussion, finding conditions granting the separability of canonical integrations becomes a very relevant problem.

The integrability of a Lie algebroid is entirely controlled by its monodromy lattices [9]. In their work on integration of bundles of Lie algebras [12], Douady and Lazard already show that separability of the canonical integration of an integrable Lie algebroid cannot be characterized by properties of the union of the monodromy lattices. In this note we describe different degrees in which separability can fail to hold, and find criteria based on properties of the union of the monodromy lattices and of the anchor map that allow us to control -partially- the lack of separability. In that respect our main results are the following propositions (see section 2 for definitions

Received by the editors April, 29, 2009. 
and recall that separability is equivalent to the submanifold of units of the groupoid being closed).

Proposition 1. Let $A \rightarrow M$ be an integrable Lie algebroid with canonical integration $\mathcal{G}(A) \rightrightarrows M$, and let $\mathcal{Z}^{0} \subset \mathcal{G}(A)$ (resp. $z \subset A$ ) be the union over the points of $M$ of the connected components of the centers of the isotropy groups (resp. of the centers of the isotropy Lie algebras). Then the submanifold of units of $\mathcal{G}(A)$ is a closed subset of $\mathcal{Z}^{0}$ if and only if the union of monodromy lattices is a closed subset of $z$.

Proposition 2. Let $A \rightarrow M$ be an integrable Lie algebroid with canonical integration $\mathcal{G}(A) \rightrightarrows M$, and let $\left\{x_{n}\right\}_{n \in \mathbb{N}} \subset M$ be a converging sequence with limit $x$. Let $G_{x} \subset$ $\mathcal{G}(A)$ denote the isotropy group over $x$ and $\exp \left(\mathfrak{g}_{x}\right) \subset G_{x}$ the image of the exponential map. Assume that for every $x_{n}$ the anchor map $\rho: A_{x_{n}} \rightarrow T_{x_{n}} M$ is injective. Then the only accumulation point of the sequence of units $\left\{1_{x_{n}}\right\}$ in $\exp \left(\mathfrak{g}_{x}\right)$ is $1_{x}$.

The homotopy groupoid of a regular foliation is separable if and only if there are no vanishing cycles [2]. For regular Poisson manifolds without vanishing cycles proposition 1 recovers a result of Alcalde-Cuesta and Hector characterizing the separability of the canonical integration [1].

Our Lie theoretic constraints in propositions 1 and 2 have also applications to singular Poisson geometry and generalized complex geometry.

Theorem 1. If $(M, \pi)$ is either a real surface with a bi-vector field or a real four manifold with a Poisson structure underlying a generalized complex structure of even type (e.g. a holomorphic bi-vector field), then the canonical integration is a Hausdorff Lie groupoid.

Theorem 1 has the following important implication.

Corollary 1. If $\pi$ is any bi-vector field on $\mathbb{R}^{2}$ then the canonical integration is diffeomorphic to $\mathbb{R}^{4}$.

In particular theorem 1 and corollary 1 extend results by Cattaneo and Felder [5] proven for planar Poisson structures with simply connected symplectic leaves.

The canonical integration of any integrable Poisson structure carries a canonical multiplicative symplectic structure [5]. The relevant question to be clarified is whether the symplectic $\mathbb{R}^{4}$ 's coming from corollary 1 are exotic or not. We plan to address this question in [13].

\section{Dividing the separability problem}

Let $\mathcal{G} \rightrightarrows M$ be any Lie groupoid with Lie algebroid $A \rightarrow M$. We introduce some notation. For each $x \in M$ we let $G_{x}$ be the isotropy group over $x, G_{x}^{0}$ the connected component of the identity, $Z_{x}$ the intersection of the center with $G_{x}^{0}, Z_{x}^{0}$ the connected component of the identity of the center, $1_{x}$ the identity, and $z_{x}$ the center of the Lie algebra of $G_{x}$. By taking union over the points of $M$ we obtain the subsets of $\mathcal{G}$

$$
\mathcal{S}:=\bigcup_{x \in M} G_{x}, \mathcal{S}^{0}:=\bigcup_{x \in M} G_{x}^{0}, \mathcal{Z}:=\bigcup_{x \in M} Z_{x}, \mathcal{Z}^{0}:=\bigcup_{x \in M} Z_{x}^{0}, M^{u}:=\bigcup_{x \in M} 1_{x}
$$

and the subset of $A$

$$
z:=\bigcup_{x \in M} z_{x}
$$


We have the inclusions

$$
\begin{gathered}
M^{u} \subset \mathcal{Z}^{0} \subset \mathcal{Z} \subset \mathcal{S}^{0} \subset \mathcal{S} \subset \mathcal{G}, \\
z \subset A,
\end{gathered}
$$

and on each subset we put the induced topology from $\mathcal{G}$ and $A$ respectively.

Because in a groupoid the multiplication and inversion are continuous, $\mathcal{G}$ is Hausdorff if and only if the submanifold of units $M^{u} \subset \mathcal{G}$ is closed. Because the source and target maps are continuous the limit of a converging sequence of units lies in $\mathcal{S}$. Therefore $\mathcal{G}$ is Hausdorff if and only if the units are closed in $\mathcal{S}$.

We want to organize as much as possible those arrows (points of $\mathcal{G}$ ) which destroy Hausdorffness. We fix a converging sequence $\left\{1_{x_{n}}\right\}_{n \in \mathbb{N}}$ of units with limit $1_{x}$. For that fixed sequence we define

$$
H=\left\{h \in G_{x} \mid h=\lim 1_{x_{n}}\right\} .
$$

Lemma 1. $H$ is a discrete subgroup of $G_{x}$.

Proof. It is a subgroup because multiplication is continuous.

The discreteness is a standard fact about limits of a fixed sequence in a manifold: If $h$ is one such point we take a chart about it. The induced topology is the one in Euclidean space where convergent sequences have just one limit, so $h$ is open in $H$. In a manifold all points are closed.

Our line of reasoning to prove separability is finding conditions that grant some extra structure on $H$, and use that structure to show that $H$ can be pushed towards the left in the chain of inclusions in (1).

Let us look at the inclusion $\mathcal{Z} \subset \mathcal{S}^{0}$ : because $H$ is discrete, then if it is already in $\mathcal{S}^{0}$ it lies in $\mathcal{Z}$ if and only if it is normal. A strategy to deduce normality would be for any fixed $g \in G_{x}^{0}$ to take a sequence of arrows $\left\{g_{n}\right\}_{n \in \mathbb{N}}$, so that (i) $\lim g_{n}=g$ and (ii) $\underline{s}\left(g_{n}\right)=x_{n}$. Then $g h g^{-1}=\lim 1_{\underline{t}\left(g_{n}\right)}$. But there is no reason in principle why the group $H$ should be not sensitive to moving each $x_{n}$ within the leaf $\mathcal{O}_{x_{n}}$ of the characteristic foliation to which the point belongs. One can check that this independence is equivalent to the possibility of choosing each $g_{n}$ inside the corresponding isotropy group for any sequence of units in the fixed sequence of leaves $\mathcal{O}_{x_{n}}$. It would be interesting to find a Lie groupoid $\mathcal{G}$ and a sequence of units for which $H$ has points in $\mathcal{S}^{0} \backslash \mathcal{Z}$

Normality of $H$ holds for bundles of Lie groups or more generally for regular Lie groupoids.

That in general $H$ cannot be pushed from $\mathcal{Z}$ into $\mathcal{Z}^{0}$ follows from [12], question 3 and remark 4. These are two examples of bundles of Lie algebras for which the canonical integration and all integrations respectively have accumulation points of the units in $\mathcal{Z} \backslash \mathcal{Z}^{0}$.

Fortunately, and because for any Lie group the connected component of the center containing the identity can be nicely controlled by the exponential map, we find a way to characterize when $H \cap \mathcal{Z}^{0}=1_{x}$. We will do it when $\mathcal{G}=\mathcal{G}(A)$ is the canonical integration because there the criterium is explicit enough to allow applications. We recall some notions and notation from [9]. 
The canonical integration is constructed as a leaf space $q: \mathcal{P}(A) \rightarrow \mathcal{G}(A)$ (with the quotient topology). The points in the Banach manifold $\mathcal{P}(A)$ are (say smooth) Apaths $a: I \rightarrow A$, and the leaves of the foliation are equivalence classes of $A$-homotopic $A$-paths.

For each $x \in M$ the monodromy lattice $N_{x} \subset z_{x}$ is the subgroup of constant $A$ paths over $x$, which are $A$-homotopic to the zero $A$-path. It is contained in the center of the isotropy Lie algebra $\mathfrak{g}_{x}$.

Consider the injective map

$$
i: z \hookrightarrow \mathcal{P}(A)
$$

sending each vector in $z$ to the corresponding constant $A$-path. We compose it with the projection $q: \mathcal{P}(A) \rightarrow \mathcal{G}(A)$, to obtain a map

$$
\exp : z \rightarrow \mathcal{Z}^{0} \subset \mathcal{G}(A) \text {. }
$$

By construction

$$
N_{x}:=\exp ^{-1}\left(1_{x}\right)
$$

We let $\mathcal{N}$ denote the union over the points of $x$ of the monodromy lattices.

Proof of proposition 1. We consider in $z \subset A$ the induced topology. The inclusion in (3) is clearly a homeomorphism over its image. Because the projection $q$ is open, exp in (4) is continuous and open. By its very construction (see also [9])

$$
\exp \left(z_{x}\right)=\mathcal{Z}_{x}^{0}
$$

Therefore $\exp : z \rightarrow \mathcal{Z}^{0}$ is onto. We form the quotient topological space $z / \mathcal{N}$, where two vectors on the same fiber are identified if and only if their difference is in the corresponding monodromy lattice. We have an induced quotient map

$$
\widetilde{\exp }: z / \mathcal{N} \rightarrow \mathcal{Z}^{0} \text {. }
$$

Because exp is open the quotient map is continuous and open, and it is also bijective by construction. Thus $\widetilde{\exp }$ is a homeomorphism. As a consequence the subset of units $M^{u}$ is closed in $\mathcal{Z}^{0}$ if and only if $\widetilde{\exp }^{-1}\left(M^{u}\right)$ is closed in $z / \mathcal{N}$. Because the latter is a quotient space this is equivalent to $\exp ^{-1}\left(M^{u}\right)$ being closed in $z$. By equation (5) $\mathcal{N}=\exp ^{-1}\left(M^{u}\right)$, and this proves the proposition.

Remark 1. We stress the fact that the criterium in proposition 1 asks for the closedness of $\mathcal{N}$ inside of $z$, not inside $A$. It is a much weaker condition, but the price to pay is that it is more difficult to apply: to see that recall that the canonical integration of the linear Poisson structure on the dual of a semisimple Lie algebra of compact type $\mathfrak{g}$ is clearly Hausdorff. If we take an orbit type and a 2-sphere generating a cohomology class, and push it towards the origin along a ray in the leaf space, any lift to $\mathfrak{g}^{*}$ gives rise to a constant path in $\mathcal{N} \subset T^{*} \mathfrak{g}^{*}=\mathfrak{g} \times \mathfrak{g}^{*}$. Over the origin it converges to a non-constant vector, so the union of the monodromy lattices is not closed in A. But it is closed in $z$ since over the origin in $\mathfrak{g}^{*}$ it reduces to the zero vector in $\mathfrak{g}$.

Proposition 1 gives the following result:

Corollary 2. If $\mathcal{N}$ coincides with the zero section then the units are closed in $\mathcal{Z}^{0}$. In particular this is the case for the canonical integration of Lie algebroids whose anchor map is injective in a dense subset [9]. 
Our second infinitesimal criterium controls lack of separability for sequences of points with injective anchor map.

Proof of proposition 2. Let $g \in \exp \left(\mathfrak{g}_{x}\right) \cap H$. Let $\left\{a_{n}\right\}_{n \in \mathbb{N}}$ be a sequence of $A$-paths representing $\left\{1_{x_{n}}\right\}$ and converging in $C^{1}$-norm to the $A$-path $a$ representing $g$; we let $\left\{\gamma_{n}\right\}_{n \in \mathbb{N}}$ denote the sequence of base paths. Homeomorphisms on $\mathcal{P}(A)$ act transitively on representatives of any class on $\mathcal{G}(A)$, so we can take $a$ to be any representative of $g$; because $g \in \exp \left(\mathfrak{g}_{x}\right)$ we can assume without loss of generality that $a$ is a constant $A$-path. We use local sections $e_{1}, \ldots, e_{d}$ to trivialize $A$ near $x$, so that $a=\left(a_{1}, \ldots, a_{d}\right)$. We also fix coordinates $y_{1}, \ldots, y_{m}$ about $x$, and we assume that all base paths are inside the domain of the local coordinates.

We must show $a=0$, and we will do it componentwise. The very elementary key observation is that because $a$ is a constant path, for any choice $\left\{t_{n}\right\} \in I$ we have

$$
a=\lim a_{n}\left(t_{n}\right) .
$$

Let $v_{n, j}=\rho_{x_{n}}\left(e_{j}\right)$. The $C^{1}$-function

$$
\begin{aligned}
S^{1} & \longrightarrow \mathbb{R} \\
t & \longmapsto\left\langle\gamma_{n}(t), v_{n, j}\right\rangle
\end{aligned}
$$

has at least two critical points (the bracket standing for Euclidean inner product). We let $t_{n, j}$ be one of them. By injectivity of the anchor we have

$$
a_{n}\left(t_{n, j}\right)=\rho^{-1}\left(\gamma_{n}^{\prime}\left(t_{n, j}\right)\right),
$$

and therefore

$$
a_{n, j}\left(t_{n, j}\right)=0
$$

this proving the result.

Next we move into finding conditions that ensure that we can push $H$ from $\mathcal{S}$ into $\mathcal{S}^{0}$.

For a (possibly singular) foliation we define a vanishing cycle $\gamma$ in a leaf $\mathcal{O}$ to be a loop non-trivial in $\pi_{1}(\mathcal{O}, x)$ which is the $C^{1}$-limit of leafwise null-homotopic loops. For a regular foliation this is the same as the classical notion of vanishing cycle [2]. A sufficient condition for the absence of vanishing cycles is asking every leaf $\mathcal{O}$ to have a saturated neighborhood $\mathcal{U}_{\mathcal{O}}$ and a retraction $r: \mathcal{U}_{\mathcal{O}} \rightarrow \mathcal{O}$ onto the leaf.

Lemma 2. Let $A$ be an integrable Lie algebroid whose underlying foliation does not have vanishing cycles. Then $\mathcal{G}(A)$ is Hausdorff if and only if $\mathcal{S}^{0}$ is Hausdorff.

Proof. One implication is obvious. The other one uses the homotopy long exact sequence for $\underline{t}: \underline{s}^{-1}(x) \rightarrow \mathcal{O}_{x}, \underline{t}, \underline{s}$ target and source maps respectively, to conclude that the absence of vanishing cycles is equivalent to $\mathcal{S}(A)$ being closed. The 1connectedness of the $\underline{s}$-fiber is needed.

We can now recover the following result of Alcalde-Cuesta and Hector:

Theorem 2 ([1]). Let $(M, \pi)$ be a regular Poisson manifold whose symplectic foliation has no vanishing cycles. Then the canonical integration is a Hausdorff Lie groupoid if and only if the union of monodromy lattices $\mathcal{N}$ is closed in $A$. 
Proof. By lemma 2 accumulation points of units are contained in $\mathcal{S}^{0}$. Because the Poisson manifold is regular all isotropy Lie algebras are abelian, so $\mathcal{S}^{0}=\mathcal{Z}^{0}$. Then proposition 1 gives the desired result.

\section{Applications and further questions}

Our application to singular Poisson geometry requires knowledge of the possible isotropy Lie algebras, so we can exclude that units accumulate out of $\mathcal{Z}^{0}$ and the image of the exponential map.

Proof of theorem 1. Singular leaves are points, and by the long exact sequence for the $\underline{s}$-fiber the isotropy group is connected. The isotropy Lie algebra at a singular point is either abelian or the Lie algebra $\mathfrak{a f f}(1)$ of the group of affine motions of the line.

Because leaves of the characteristic foliation are either open symplectic subsets or points, the monodromy lattice is the zero section, so by corollary 2 the units do not accumulate on $\mathcal{Z}^{0} \backslash M^{u}$. Therefore separability may only fail for sequences of units converging to a unit over a singular point whose isotropy lie algebra is $\mathfrak{a f f}(1)$. Because about these points there is a normal form for the Poisson tensor $[15,7]$ (the normal form up to $B$-transform for generalized complex structures of even type in a neighborhood of a non-degenerate singular point implies a normal form for the underlying Poisson tensor), if the $\left\{x_{n}\right\}_{n \in N}$ are critical points then all $A$-paths can be seen as $A$-paths inside the isotropy Lie algebra over the limit point, so they converge to the unit. If the the $\left\{x_{n}\right\}_{n \in N}$ are regular, because exp: $\mathfrak{a f f}(1) \rightarrow \mathfrak{A f f}(1)$ is onto proposition 2 implies that the limit is the unit, and thus we conclude that $\mathcal{G}(A)$ is Hausdorff.

Remark 2. Theorem 1 implies that for real surfaces the canonical integration is a (separable) symplectic 4-manifold.

For generalized complex structures of even type on a four manifold, the canonical integration is a (separable) eight dimensional symplectic manifold with a $(1,1)$ tensor fitting into a Hitchin groupoid [8]; if the generalized complex structure came from a holomorphic Poisson structure then the canonical integration is a (separable) holomorphic symplectic 4-fold. It might be interesting to interpret the results of [7] at the level of the canonical integration, and explore whether something can be added by using the groupoid approach.

It is important to advance further in the description of canonical integrations. Separability plays a key role in the following result:

Corollary 3. If $(M, \pi)$ is a real surface with a bi-vector field then the source map makes the canonical integration into a locally trivial fibration over $M$.

Proof. A result of Meigniez [14] implies that any submersion whose fibers are abstractly diffeomorphic to a Euclidean space and whose total space is Hausdorff, is locally trivial.

Corollary 1 is a straightforward consequence of corollary 3 . When the surface is not the plane but we know a bit more about the Poisson structure, it is still possible to fully describe the canonical integrations as manifolds. In particular for topologically 
stable Poisson structures on an orientable surface $\Sigma$ as defined by Radko [15], the canonical integration is diffeomorphic to $\mathbb{R}^{2} \times \Sigma[13]$.

According to remark 2 the holomorphic Poisson structure defined on a smooth toric surface with anti-canonical divisor of points with non-trivial isotropy for the action, integrates into a separable holomorphic symplectic (non-compact) 4 -fold. It should be possible to describe this 4 -fold in terms of the combinatorial data defining the toric variety.

\section{Acknowledgements}

I would like to thank Marius Crainic for Rui Loja Fernandes for many stimulating discussions about the topic of this paper; I am also grateful to the referee for his/her valuable suggestions. I would like to acknowledge financial support from NWO Open Competitie Project 613.000.425, and the Fundação para a Ciência e a Tecnologia (FCT /Portugal) Project POCTI 2010/FEDER.

\section{References}

[1] F. Alcalde-Cuesta, G. Hector, Intégration symplectique des variétés de Poisson régulières. (French) [Symplectic integration of regular Poisson manifolds] Israel J. Math. 90 (1995), no. $1-3,125-165$.

[2] F. Alcalde Cuesta, G. Hector, Feuilletages en surfaces, cycles évanouissants et variétés de Poisson. (French) [Surface foliations, vanishing cycles and Poisson manifolds] Monatsh. Math. 124 (1997), no. 3, 191-213.

[3] C. Arias, M. Crainic, The Weil algebra and the Van Est isomorphism. Preprint arXiv:0901.0322.

[4] H. Bursztyn, M. Crainic, A. Weinstein, C. Zhu, Integration of twisted Dirac brackets. Duke Math. J. 123 (2004), no. 3, 549-607.

[5] A. Cattaneo, G. Felder, Poisson sigma models and symplectic groupoids. Quantization of singular symplectic quotients. 61-93, Progr. Math., 198, Birkhäuser, Basel, 2001.

[6] A. Cattaneo, P. Xu, Integration of twisted Poisson structures. J. Geom. Phys. 49 (2004), no. 2, $187-196$

[7] G. Cavalcanti, M. Gualtieri, Blow-up of generalized complex 4-manifolds. Preprint arXiv:0806.0872.

[8] M. Crainic, Generalized complex structures and Lie brackets. Preprint math.DG/0412097.

[9] M. Crainic, R.L. Fernandes, Integrability of Lie brackets. Ann. of Math. (2) 157 (2003), no. 2, $575-620$

[10] M. Crainic, R.L. Fernandes, A geometric approach to Conn's linearization theorem. Preprint arXiv:0812.3060

[11] M. Crainic, C. Zhu, Integrability of Jacobi and Poisson structures. Ann. Inst. Fourier (Grenoble) 57 (2007), no. 4, 1181-1216.

[12] A. Douady, M. Lazard, Espaces fibrés en algèbres de Lie et en groupes. (French) Invent. Math. 1 (1966) 133-151.

[13] D. Martínez Torres, On the canonical integration of topologically stable Poisson structures. In preparation.

[14] G. Meigniez, Submersions, fibrations and bundles. Trans. Amer. Math. Soc. 354 (2002), no. 9, $3771-3787$.

[15] O. Radko, A classification of topologically stable Poisson structures on a compact oriented surface. J. Symplectic Geom. 1 (2002), no. 3, 523-542.

Centro de Análise Matemática, Geometria e Sistemas Dinâmicos, Departamento de Matemática, Instituto Superior Técnico, Av. Rovisco Pais, 1049-001 Lisboa, Portugal

E-mail address: martinez@math.ist.utl.pt 